\title{
Synthesis 1,2-bis ((3-methylbut-2-en-1-yl)oxy) -4-(((3methylbut-2-en-1-yl)oxy)methyl)benzene
}

\author{
Ahmad Rizqi Alima Fabri and Mardi Santoso \\ Department of Chemistry, Institut Teknologi Sepuluh Nopember, Surabaya \\ e-mail: tsv09@chem.its.ac.id
}

\begin{abstract}
Abstrak - Kojic acid and arbutin are materials that are used to inhibit melanogenesis, but in their development, these two compounds have an adverse effect on the skin. 3,4dihydroxybenzaldehyde is a simple phenol compound whose derivatives are used as flavorers and fragrance. The research carried out aims to obtain 3,4-dihydroxybenzaldehyde derivatives in the form of 1,2-bis((3-methylbut-2-en-1-yl)oxy) 4(((3-methylbut-2-en-1-yl)oxy)methyl)benzene which is estimated based on its structure is able to inhibit melanogenesis, and has a similar structure to the commercial fragrance of methyl diantilis. The synthesis of the target compound is carried out in three step. The first step involves the alkylation of 3,4dihydroxybenzaldehyde with 3,3-dimethylalyl bromide and sodium hydride in the dimethylformamide solvent at room temperature to obtain 3,4-bis ((3-methylbut-2-en-1-il) benzaldehyde. Reduction of formyl 3,4-bis((3-methylbut-2-en-1il) benzaldehyde which is carried out in the second stage with sodium borohydride in ethanol obtained 3,4-bis((3-methylbut-2en-1-yl)oxy)phenyl)methanol. Etherification 3,4-bis ((3methylbut-2-en-1-il)oxy)phenyl)methanol with 3,3-dimethylalyl bromide and sodium hydride in the solvent dimethylformamide at room temperature is obtained target compound 1,2-bis $((3-$ methylbut-2-en-1-il)oxy)-4-(((3-methylbut-2-en-1

il)oxy)methyl)benzene whose structure is determined by NMR, mass, and IR spectroscopy.
\end{abstract}

Kata Kunci-Synthesis, 3,4-Dihydroxybenzaldehyde Derivatives, Melanogenesis.

\section{INTRODUCTION}

$\mathrm{H}$ AVING healthy skin is what everyone wants, especially women. Cosmetics is one way of skincare, which among others plays a role in preventing the occurrence of hyperpigmentation [1]. Kojic acid (1) and arbutin (2) are used to inhibit hyperpigmentation, but in its development, these two compounds have an impact on the skin. Arbutin (2) can cause allergies, and long-term use will worsen hyperpigmentation, whereas kojic acid (1) can cause skin irritation [1], [2]. Arung et al (2007) [3] succeeded in separating several compounds (3) from jackfruit plants (Artocapus heterophyllus) which have activity as melanogenesis inhibitors. The three compounds can be seen in Figure 1. The compounds (3) separated from the plant were tested for their bioactivity against melanoma B16 cells with kojic acid (1) and arbutin (2) as positive controls. The results can be seen in Table 1 . Table 1 shows that in general compounds (3a-f) have better melanogenesis inhibiting activities than kojic acid (1) and arbutin (2) against melanoma B16 cells. The data shows that the presence of 3-methyl-2butenyl groups plays a role in inhibiting melanogenesis as can be seen in the $\mathrm{IC}_{50}$ value, the more 3-methyl-2-butenyl groups can increase their activity in inhibiting melanogenesis.
3,4-Dihydroxybenzaldehyde is a simple phenol compound. Derivatives of 3,4-dihydroxybenzaldehyde are used as flavorings and deodorizers, for example, 4-hydroxy-3methoxybenzaldehyde, 3,4-dimethoxybenzaldehyde and 1,3benzaldioxol-5-carboxaldehyde [4] Methyl dianthilis (4), which was first introduced by the leading company, perfume and flavor Givaudan from Switzerland, has a spicy, carnation, sweet, vanilla aroma. Methyl methylated (4) is used as a substitute for isoeugenol (5) (see Figure 2) in the use of shampoo and perfume [5].

Literature studies that have been carried out provide inspiration to synthesize compound (6) which is designed to have activity as an inhibitor of melanogenesis and has a similar structure to methyl dianthilis. The synthesis of the target compound (6) was suggested by a retrosynthetic analysis approach as can be seen in Figure 3.

The research carried out aims to obtain 1,2-bis((3methylbut-2-en-1-il)oxy)-4-((3-methylbut-2-en-1-

il)oxy)methyl)benzene (6) with the basic ingredients of 3,4dihydroxy benzaldehyde, using a retrosynthetic analysis approach. It also found three derivatives 3,4dihydroxybenzaldehyde in the form of 1,2-bis((3-methylbut2-en-1-il)oxy)-4-((3-methylbut-2-en-1-

il)oxy)methyl)benzene (6), 3,4-bis((3-methylbut-2-en-1-iloxy)phenyl)methanol (7), 3,4-bis((3-methylbut-2-en-1il)benzaldehyde (8) (see Figure 3).

\section{METHOD}

\section{A. Tools and Materials}

The experiment was carried out using laboratory equipment as follows: goblet, Erlenmeyer, separating funnel, watch glass, weighing bottle, measuring cup, double neck flask, drop pipette, volume pipette, propipet, Socorex Swiss micropipette, magnetic stirrer hotplate, thin layer chromatography (TLC) plat aluminum silica 60 F254 (Merck 1.05554), Sartorius CP224S analytical balance, capillary pipelines, separating funnels, TLC vessels, UV lamps ( $\lambda 254$ and $366 \mathrm{~nm}$ ), Shimadzu FTIR 8400S analytical balance, HP 6890 and KP2010 Ultra gas mass spectrometers Jeol ECS NMR spectrometers (400 Mhz).

The materials used in the research included 3,4-dihydroxy benzaldehyde (Merck 8.20475), 3.3-dimethylalyl bromide (Fluka 38925), amberlyst 15 (Sigma Aldrich 216380), sodium hydride (Merck 8.14552), sodium borohydride (Merck 38925), amberlyst 1515 (Sigma Aldrich 216380), sodium hydride (Merck 8.14552), sodium borohydride (Merck 38925), amberlyst 15 (Sigma Aldrich 216380), sodium hydride (Merck 8.14552), sodium borohydride 
<smiles>O=c1cc(CO)occ1O</smiles>

(1)

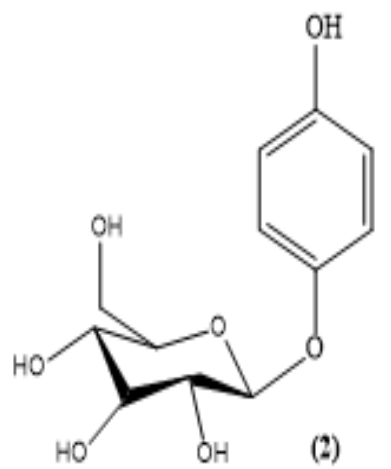



(3a-f)

Figure 1. Melanogenesis inhibiting compounds: (1) kojic acid, (2) arbutin, and (3) compounds from Artocapus heterophyllus.

Table 1.

Activities of compounds (3a-f) as inhibitors of hyperpigmentation

\begin{tabular}{|c|c|c|c|c|c|c|c|}
\hline Compound & $\overline{\mathbf{R}_{1}}$ & $\mathbf{R}_{2}$ & $\mathbf{R}_{3}$ & $\mathbf{R}_{4}$ & $\overline{\mathbf{R}_{5}}$ & $\mathbf{R}_{6}$ & $\mathrm{IC}_{50}(\mu \mathrm{M})$ \\
\hline (3a) & 3-methyl-2-butenyl & $\mathrm{OH}$ & 3-methyl-2-butenyl & $\mathrm{OMe}$ & $\mathrm{H}$ & $\mathrm{OH}$ & 7.3 \\
\hline (3b) & 3-methyl-2-butenyl & $\mathrm{OH}$ & 3-methyl-2-butenyl & $\mathrm{OH}$ & $\mathrm{H}$ & $\mathrm{OH}$ & 3.8 \\
\hline (3c) & $\mathrm{H}$ & $\mathrm{OH}$ & 3-methyl-2-butenyl & $\mathrm{OH}$ & $\mathrm{H}$ & $\mathrm{H}$ & 6.6 \\
\hline (3d) & 3-methyl-2-butenyl & $\mathrm{OH}$ & $\mathrm{H}$ & $\mathrm{OH}$ & 3-methyl-2-butenyl & $\mathrm{OH}$ & 4.9 \\
\hline (3e) & 3-methyl-2-butenyl & $\mathrm{OH}$ & $\mathrm{H}$ & $\mathrm{H}$ & $\mathrm{H}$ & $\mathrm{OH}$ & 40.1 \\
\hline (3f) & $\mathrm{H}$ & $\mathrm{OH}$ & $\mathrm{H}$ & $\mathrm{OH}$ & $\mathrm{H}$ & $\mathrm{H}$ & 32.9 \\
\hline (1) & & & & & & & $>3521$ \\
\hline (2) & & & & & & & 111 \\
\hline
\end{tabular}

(Merck No. 1,00137,2500), methanol (Fulltime-6501), ethanol (Smart Lab A-1035), ethyl acetate (ACS 6801-04), nhexane (Meck 1.04367), NN-dimethylformamide (DMF) (Merck 1.03053), dichloromethane (Merck 1.06050), Silica GF 60 gel (Merck 1.07734.1000), distilled water, magnesium sulfate heptahydrate (Merck 1.005886).

\section{B. Experiment Procedures}

1) Synthesis 3,4-bis((3-methylbut-2-en-1-il)benzaldehyde (8)

Synthesis of 3,4-bis ((3-methylbut-2-en-1-il)benzaldehyde (8) was carried out by adapting the 5,6methylenedioxyaindole methylation procedure [6]. 3,4dihydroxybenzaldehyde solution (0.156 gram; $1.128 \mathrm{mmol})$ in anhydrous DMF $(15 \mathrm{~mL})$, plus sodium hydride $(0.372$ grams; $15.506 \mathrm{mmol}$ ) and stirred at room temperature. The mixture was then placed in an ice bath and added 3.3dimethylalyl bromide $(0.800 \mathrm{~mL} ; 6.8911 \mathrm{mmol})$ dropwise while stirring at room temperature. The mixture was stirred further at room temperature, and the reaction is monitored by TLC. The organic phase obtained was combined, and washed with distilled water $(3 \times 15 \mathrm{~mL})$, dried with anhydrous magnesium sulfate, and evaporated at low pressure. The synthesized product was then purified by gravity column chromatography with silica gel GF 60 and mobile phase $n$ Hexana: ethyl acetate $(9: 1)$, purity test was carried out by 3 dimensional TLC and two-dimensional TLC. The pure synthesis results were weighed and identified by NMR spectrophotometer.

\section{2) Synthesis 3,4-bis((3-methylbut-2-en-1-}

il)oxy)phenyl)methanol (7)

Synthesis of 3,4-bis ((3-methylbut-2-en-1-il) oxy) phenyl) methanol (7) was carried out by adapting the reduction procedure 4,6-dimethoxy-1-methylindol-3-carbaldehyde [6]. 3,4-bis((3-methylbut-2-en-1-il)benzaldehyde (8) solution $(0.054$ gram; $0.196 \mathrm{mmol})$ in ethanol $(10 \mathrm{~mL})$ plus sodium borohydride $(0.034$ gram; $0.898 \mathrm{mmol})$, then stirred it at room temperature and the reaction was monitored by TLC, the reaction results were then added with a solution of sodium hydroxide $10 \%(10 \mathrm{~mL})$ after the reaction was complete, and extracted with dichloromethane $(3 \times 10 \mathrm{~mL})$. The organic phase obtained was combined, washed with distilled water $(30 \mathrm{~mL})$ ), dried with magnesium sulfate anhydrous, and evaporated at low pressure. The results of the synthesis were tested for purity by TLC and two-dimensional TLC. The pure synthesis results were weighed and identified by NMR spectrophotometer.

3) Synthesis 1,2-bis((3-methylbut-2-en-1-il)oxy)-4-((3methylbut-2-en-1-il)oxy)methyl)-benzene (6)

Synthesis of 1,2-bis((3-methylbut-2-en-1-il)oxy)-4-((3methylbut-2-en-1-il)oxy)methyl)benzene (6) was done by adapting 3,4-methylenedioxybenzyl methyl ether synthesis procedure [2]. 3,4-bis solution((3-methylbut-2-en-1il)oxy)phenyl)methanol (7) (0.041 gram; $0.148 \mathrm{mmol})$ in DMF (15 mL) plus sodium hydride (0.022 gram; $0.917 \mathrm{mmol}$ ) then stirred at room temperature. The mixture was then placed in an ice bath and added 3.3-dimethylalyl bromide 
The $6^{\text {th }}$ International Seminar on Science and Technology (ISST) 2020

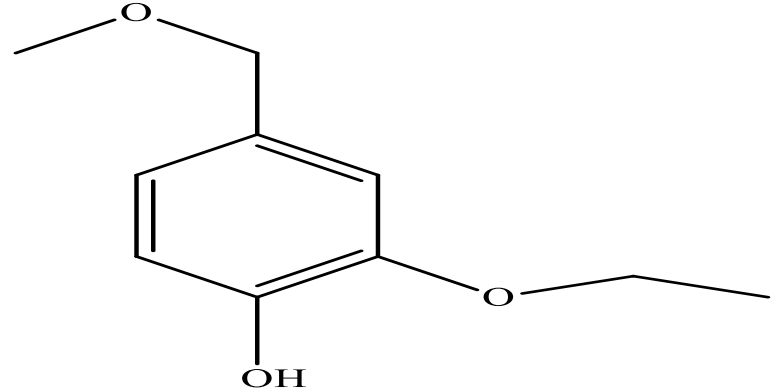

(4)

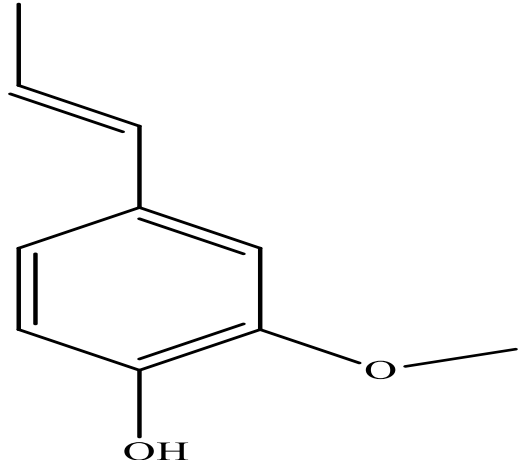

(5)

Figure 2. Compounds of methyl dianthilis (4) and isoeugenol (5).

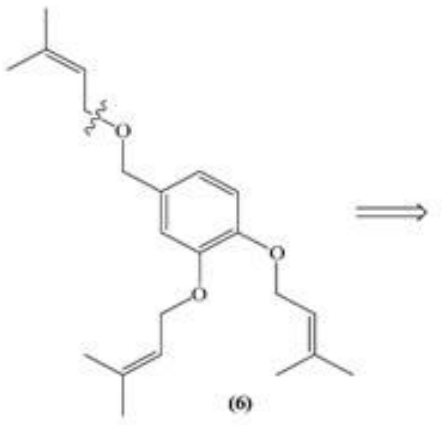

Figure 3. Retrosynthetic Analysis of Target Compounds

(0.030 $\mathrm{mL} ; 0.255 \mathrm{mmol})$ dropwise while stirring at room temperature. The mixture was stirred further at room temperature, and the ongoing reaction was monitored by TLC. The reaction mixture was added with cold distilled water $(20 \mathrm{ml})$ after the reaction was complete, and extracted with dichloromethane $(3 \times 10 \mathrm{~mL})$. The obtained organic phase was combined, and washed with distilled water (15 $\mathrm{mL}$ ), dried with anhydrous magnesium sulfate, and evaporated at low pressure. The synthesized product was then purified by gravity column chromatography with silica gel GF 60 stationary phase and $n$-hexane: ethyl acetate (10:1) mobile phase, purity test was carried out by 3 -dimensional TLC and two-dimensional TLC. The pure synthesis results were weighed and identified by IR and NMR spectrometers, and KG-SM.

\section{RESULTS AND DISCUSSION}

\section{A. Synthesis 3,4-bis((3-methylbut-2-en-1-il)benzaldehyde} (8)

Synthesis of 3,4-bis((3-methylbut-2-en-1-il)benzaldehyde (8) was carried out in two stages. The first step involved the reaction of 3,4-dihydroxybenzaldehyde with sodium hydride in DMF solvent at room temperature for 15 minutes; in the second stage the reaction results in the first stage were further reacted with 3,3-dimethylalyl bromide and the ongoing reaction was monitored by $\mathrm{TLC}$, the reaction results were then added with cold distilled water $(30 \mathrm{~mL})$, and extracted with dichloromethane $(3 \times 10 \mathrm{~mL})$. The obtained organic phase was combined, washed with distilled water $(15 \mathrm{~mL})$, dried with anhydrous magnesium sulfate, and evaporated at low pressure to obtain a clear liquid of 0.22 grams. The subsequent synthesis was purified using gravity column chromatography with silica gel GF 60 and the mobile phase of $n$-hexane: ethyl acetate (9:1) The chromatography column gravity results obtained 27 fractions, fractions 14-25 which showed a single stain then combined, evaporated at low pressure, and tested for purity by TLC.

Identification of the structure of the pure synthesized compound with an NMR spectrometer given a ${ }^{1} \mathrm{H}$ NMR spectrum. The ${ }^{1} \mathrm{H}$ NMR spectrum showed six signals from upfield to downfield with an integration ratio of $6: 6: 4: 2: 1: 2: 1$. The prenyl group gave four signals as follows: two singlet signals with a 6: 6 integration in the chemical shift $(\delta) 1.73$ and $1.76 \mathrm{ppm}$ were signals from the protons of the two methyl groups; doublet signal doublet with integration 4 at 644.64 (J 
The $6^{\text {th }}$ International Seminar on Science and Technology (ISST) 2020

July $25^{\text {th }} 2020$, Institut Teknologi Sepuluh Nopember, Surabaya, Indonesia

$=17.4 ; 6.2 \mathrm{~Hz}) \mathrm{ppm}$ is the signal of the protons of the two methylene groups, and the multiplex signal with integration 2 at $\delta$ 5.47-5.49 ppm is a signal from two protons of a metin group. A doublet signal with integration 1 at $\delta 6.94(\mathrm{~d}, \mathrm{~J}=8$ $\mathrm{Hz}) \mathrm{ppm}$ is a signal from one aromatic proton, a multiplex signal with integration 2 at $\delta 7.38-7.41 \mathrm{ppm}$ was a signal of two aromatic protons, and singlet signals with integration 1 at $\delta 9.80 \mathrm{ppm}$ were signals from protons of formal groups.

The reactions of 3,4-dihydroxybenzaldehyde $(0.156$ grams; $1.128 \mathrm{mmol}), 3,3$-dimethylalyl bromide $(0.800 \mathrm{~mL}$; $6.911 \mathrm{mmol})$, sodium hydride (0.372 gram; $15.506 \mathrm{mmol})$ in DMF solvents at room temperature resulting in 3,4-bis(3methylbut-2-en-1-il)benzaldehyde (8) as much as 0.13 grams or with a yield of $45 \%$. The reaction time required for 3,4bis synthesis(( 3-methylbut-2-en-1-il)benzaldehyde (8) was 75 minutes, which was shorter than reported by $\mathrm{Li}$ et al. (2011) [7] which takes 24 hours. Li et al. (2011) [7] used potassium carbonate as the base, and acetone as solvents Hydride ions as a base react with two groups hydroxies of 3,4-dihydroxybenzaldehyde to form anions with better nucleophility, which then react with prenyl bromide through sub-reaction mechanisms nucleophilic substitutes to form 3,4-bis ((3-methylbut-2-en-1-il)benzaldehyde (8) with byproducts in the form of hydrogen gas and sodium bromide.

\section{B. Synthesis 3,4-bis((3-methylbut-2-en-1-} il)oxy)phenyl)methanol (7)

Synthesis of 3,4-bis((3-methylbut-2-en-1il)oxy)phenyl)methanol (7) was carried out by reducing the formyl group of 3,4-bis((3-methylbut-2-en-1il)benzaldehyde $(\mathbf{8})$ with sodium borohydride carried out in ethanol at room temperature The ongoing reaction was monitored by TLC. The TLC results showed that the reaction has been completed after the reaction has lasted for 15 minutes. The reduction results were then added with a $10 \%$ sodium hydroxide solution $(10 \mathrm{~mL})$, then extracted with dichloromethane $(3 \times 10 \mathrm{~mL})$ The organic phase obtained was combined, washed with distilled water $(30 \mathrm{~mL})$, dried with anhydrous magnesium sulfate, and evaporated at low pressure to obtain the synthesis results in the form of yellow liquid as much as 0.041 grams.

The identification of the reduced structure with NMR spectrometers gives ${ }^{1} \mathrm{H}$ NMR spectrum. The ${ }^{1} \mathrm{H}$ NMR spectrum clearly showed that the singlet signal of the formal group at $\delta 9.81 \mathrm{ppm}$ was not visible, this showed that the reduction was successful. The prenyl group gave four signals as follows: two singlet signals with 6: 6 integration at 1.73 and $1.79 \mathrm{ppm}$ respectively were signals of the protons of two methyl groups; singlet signals with integration 6 at $\delta 4.95$ ppm were signals from protons of two methylene groups (including methylene protons from hydroxymethyl groups), and multiplet signals with integration 2 at $\delta 5.46-5.48 \mathrm{ppm}$ were signals from two protons of a metin group. Multiplet signal with integration 3 at pada 6,82-6,94 ppm was a signal of three aromatic protons.

Reduction of 3,4-bis((3-methylbut-2-en-1-il) enzaldehyde (8) (0.054 gram; $0.196 \mathrm{mmol})$ with sodium borohydride (0.034 gram; $0.898 \mathrm{mmol})$ in ethanol $(10 \mathrm{~mL})$ at room temperature

3.4-bis((3-methylbut-2-en-1il)oxy)phenyl)methanol (7) of 0.041 grams or with a yield of $77 \%$. The reduction mechanism of 3,4-bis((3-methylbut-2en-1-il)benzaldehyde (8) was suggested to proceed as the reduction of aldehyde groups in general [8].

\section{Synthesis of 1,2-bis((3-methylbut-2-en-1-il)oxy)-4-((3- methylbut-2-en-1-il)oxy)methyl)benzene (6)}

Synthesis of 1,2-bis((3-methylbut-2-en-1-il)oxy)-4-((3methylbut-2-en-1-il) oxy)methyl)benzene (6) was carried out in two steps. The first step involved the reaction of 3,4-bis((3methylbut-2-en-1-il)oxy)phenyl)methanol (7) with sodium hydride in the DMF solvent at room temperature for 15 minutes. The results of the reaction in the first step were further reacted with 3,3-dimethylalyl bromide (second step). The reactions that take place in the second stage were monitored by TLC. The results of monitoring the reactions that took place at minutes 15,30 , and 60 there were no significant changes; the reaction product was then added with cold distilled water $(20 \mathrm{~mL})$ and extracted with dichloromethane $(3 \times 10 \mathrm{~mL})$. The organic phase obtained was combined, washed with distilled water $(15 \mathrm{~mL})$, dried with anhydrous magnesium sulfate, and evaporated at low pressure so that a clear liquid of 0.043 gram is obtained.

The synthesized product was further purified by gravity column chromatography with silica gel GF stationary phase and $n$-hexane: ethyl acetate (10:1) mobile phase. Fractions 25-31 which showed a single stain were then combined, evaporated at low pressure, and tested for purity by TLC. Analysis of the results of the reaction by gas chromatography mass spectrometers showed a major peak with a retention time of 32.836 minutes. The spectrum showed the peak of the molecular ion at $m / z 344.2$ which corresponds to the relative mass of 1,2-bis((3-methylbut-2-en-1-il)oxy)-4-((3methylbut-2-en -1-il)oxy)metl)-benzene (6). The compound molecule ion (6) released successive isoprene molecules so that fragments with peaks at $m / z 276,208$, and 140 are produced. Fragments with peaks at $m / z 208$ then release 3methyl-2-butenal molecules resulting in fragments with a peak at $\mathrm{m} / \mathrm{z} 124$ (base peak). Release of hydrogen gas from a fragment with a peak at $\mathrm{m} / \mathrm{z} 140$ produced fragments with a peak at $\mathrm{m} / \mathrm{z}$ 138. Molecular ion compounds (6) also undergo fragmentation to form prenyl cations with peaks at $m / z 69$ which subsequently release etuna and hydrogen to form cations with peaks at $m / z, 43$ and 41 .

Further identification of the structure of the synthesized compound with an NMR spectrometer gives a ${ }^{1} \mathrm{H}$ NMR spectrum. The ${ }^{1} \mathrm{H}$ NMR spectrum showed ten signals from the upfield to downfield in a ratio of $3: 3: 3: 6: 3: 2: 2: 4: 1: 3$. The prenil group gave six signals as follows: five singlet signals with $3: 3: 3: 6: 3$ integration on chemical shift $(\delta) 1.65 ; 1.71$; $1.72 ; 1.75$ and $1.76 \mathrm{ppm}$ respectively are signals from the protons of the methyl group; two doublet signals with 2: 4 integration at $\delta 3.97(\mathrm{~J}=6.8 \mathrm{~Hz})$ and $4.58(\mathrm{~J}=6.8 \mathrm{~Hz}) \mathrm{ppm}$ respectively were signals of two and four protons from the methylene group coupled with the methane proton ; and two triplet signals with 1:2 integration at $\delta 5.39(\mathrm{~J}=1.2 \mathrm{~Hz})$ and $5.50(\mathrm{~J}=6.4 \mathrm{~Hz}) \mathrm{ppm}$ respectively were signals of one and two protons from the proton-coupled metin group -proton 
The $6^{\text {th }}$ International Seminar on Science and Technology (ISST) 2020

July $25^{\text {th }} 2020$, Institut Teknologi Sepuluh Nopember, Surabaya, Indonesia

methylene group. The ${ }^{1} \mathrm{H}$ NMR spectrum also showed a singlet signal with integration 2 at 424.42 ppm which was a signal of two protons from the methylene benzyl group; and multiplet signals with the integration of $3 \delta 6.83-6.91 \mathrm{ppm}$ were signals of three aromatic protons.

This identification was strengthened by the ${ }^{13} \mathrm{C}$ NMR spectrum of 1,2-bis((3-methylbut-2-en-1-il)oxy)-4-((3methylbut-2-en-1-il)oxy)methyl)benzene (6), which showed 21 signals. Signals at $\delta 18.17 ; 18.33 ; 25,90 ; 25.93$ ppm was a signal of carbon-metal metal groups of three prenyl groups. The carbons of the methylene group give signals at 9465.94 ; $66.14 ; 66.34$; and 72.11 ppm. Signals at $\delta 120.33 ; 120.46$; and $120.58 \mathrm{ppm}$ were the signals of three methane carbons from three prenyl groups; and signals at $\delta 137.11 ; 137.23$, and $137.27 \mathrm{ppm}$ were quaternary carbon signals from three prenyl groups. Aromatic carbons gave signals at $\delta 113.70 ; 113.74$; $121.21 ; 131.27 ; 148.32$; and $148.94 \mathrm{ppm}$.

The reaction of 3,4-bis((3-methylbut-2-en-1il)oxy)phenyl)methanol (7) (0.041 gram; $0.148 \mathrm{mmol}), 3.3$ dimethylalyl bromide $(0.030 \mathrm{~mL} ; 0.255 \mathrm{mmol})$, and sodium hydride $(0.022$ gram; $0.917 \mathrm{mmol})$ obtained 1,2-bis((3methylbut-2-en-1-il)oxy)-4-((3-methylbut-2-en-1-

il)oxy)methyl)-benzene (6) 0.013 grams or with a yield of $26 \%$. The hydride ion as a base reacts with a hydroxy group of 3,4-bis ((3-methylbut-2-en-1-il) oxy) phenyl) methanol (7) to form anions with better nucleophility, which then reacts with prenyl bromide through the nucleophilic substitution reaction mechanism to form 1,2-bis((3-methylbut-2-en-1il)oxy)-4((3-methylbut-2-en-1-il)oxy)methyl)-benzene (6) with the byproducts in the form of hydrogen gas and sodium bromide.

\section{CONLUSION}

The reaction of 3,4-dihydroxybenzaldehyde, 3,3dimethylalyl bromide, sodium hydride in DMF solvent at room temperature resulted in 3,4-bis((3-methylbut-2-en-1- il)benzaldehyde (8) with a yield of $45 \%$ The reduction of 3,4bis((3-methylbut-2-en-1-il)benzaldehyde (8) with sodium borohydride in ethanol at room temperature obtained 3,4bis((3-methylbut-2-en-1-il)oxy)phenyl)methanol (7) with $77 \%$ yield 3,4-bis((3-methylbut-2-en-1il)oxy)phenyl)methanol (7), 3,3-dimethylalyl bromide, and sodium hydride obtained 1,2-bis((3-methylbut-2-en-1yl)oxy)-4-((3-methylbut-2-en-1-yl) oxy)methyl)-benzene (6) with a yield of $26 \%$.

\section{ACKNOWLEDGMENT}

The authors thank to the Laboratory of Natural Product and Synthesis Chemistry, Department of Chemistry, Faculty of Science, Institut Teknologi Sepuluh Nopember.

\section{REFERENCES}

[1] P. E. Grimes, "Management of Hyperpigmentation in Darker Racial Ethnic Groups," Seminars in Cutaneous Medicine and Surgery, vol. 28, no. 2. Semin Cutan Med Surg, pp. 77-85, Jun-2009.

[2] K. Ishida and E. Tamai, "Melanogenesis inhibitor and skin preparation containing the same," US7576125B2, 12-Feb-2009.

[3] E. T. Arung, K. Shimizu, and R. Kondo, "Structure-Activity Relationship of Prenyl-Substituted Polyphenols fromArtocarpus heterophyllus as Inhibitors of Melanin Biosynthesis in Cultured Melanoma Cells," Chemistry \& Biodiversity, vol. 4, no. 9, pp. 21662171, Sep. 2007.

[4] A. Braga, C. Guerreiro, and I. Belo, "Generation of Flavors and Fragrances Through Biotransformation and De Novo Synthesis," Food and Bioprocess Technology, vol. 11, no. 12, pp. 2217-2228, Dec. 2018.

[5] W. H. Miles and K. B. Connell, "Synthesis of Methyl Diantilis, a commercially important fragrance," Journal of Chemical Education, vol. 83, no. 2, pp. 285-286, Feb. 2006.

[6] M. Santoso, K. Somphol, N. Kumar, and D. S. C. Black, "Synthesis of indolocyclotriveratrylenes," Tetrahedron, vol. 65, no. 31, pp. 59775983, Aug. 2009.

[7] J. Li et al., "Studies on synthesis and structure-activity relationship (SAR) of derivatives of a new natural product from marine fungi as inhibitors of influenza virus neuraminidase," Marine Drugs, vol. 9, no. 10, pp. 1887-1901, 2011.

[8] F. A. Carey, Organic Chemistry, 4th Edition,. Virginia, 2000. 